Portsmouth University

Relativity and Cosmology Group

Preprint RCG 95/9

\title{
Quadrupole anisotropy from photon quantum effects
}

\author{
Alexei V. Nesteruk[ and Roy Maartens ${ }^{1} \cdot{ }^{[}$
}

\begin{abstract}
We consider quantum effects of an electromagnetic field in a radiation-dominated almost FRW spacetime. The dominant non-local quantum correction to the photon distribution is a quadrupole moment, corresponding to an effective anisotropic pressure in the energy-momentum tensor.
\end{abstract}

\section{Introduction}

Anisotropy in a cosmological photon distribution usually refers to the cosmic background radiation after the decoupling of photons from matter. Such anisotropy is predominantly caused by density perturbations in the matter at the epoch of last scattering [1]. Here we investigate another kind of anisotropy, not connected with density perturbations or gravity wave perturbations, and not arising from scalar field fluctuations in the inflationary epoch, but arising from vacuum-polarisation type quantum effects of the electromagnetic field itself.

We are only able to treat these effects in the case of a field that is non-interacting with other matter fields. Therefore the treatment is only really applicable to the radiation-dominated epoch. These early-universe effects would be washed out by the interaction of photons with matter during the recombination epoch. As such, our

\footnotetext{
${ }^{1}$ School of Mathematical Studies, Portsmouth University, PO1 2EG England

${ }^{2}$ Member of Centre for Nonlinear Studies, Witwatersrand University, South Africa
} 
results are a contribution not to the theory of the cosmic background radiation, but to quantum field theory in curved spacetime and to early-universe dynamics.

We consider an early universe with small inhomogeneities and anisotropies, i.e. with an almost Friedmann-Robertson-Walker (FRW) geometry. In order to obtain a stable vacuum state for massless fields and to avoid infinite creation of particles in the vicinity of the singularity $t=0$, we impose the Weyl curvature hypothesis of Penrose [2], i.e. that the Weyl curvature should tend to zero as the singularity is approached. Thus asymptotically, as $t \rightarrow 0$, the spacetime becomes FRW. In order to invoke standard results of quantum field theory, we further assume that the spacetime is spatially homogeneous, so that the (small) deviations from FRW geometry are only anisotropies. This is not unreasonable for the early universe. Indeed, the anisotropies can be made as small as desired, provided that they do not vanish exactly. Nonvanishing anisotropy in the spacetime gives rise to the quantum effects that produce effective anisotropy in the photon distribution (quantum field theory is not analytic in the parameter describing the degree of anisotropy).

Since we are primarily concerned with demonstrating the existence of these effects in principle, we will simplify the calculations by assuming that the anisotropies are of Bianchi I type, so that the metric has the form

$$
d s^{2}=g_{\mu \nu} d x^{\mu} d x^{\nu}=-d t^{2}+A_{i}^{2}(t)\left(d x^{i}\right)^{2}
$$

where $i=1,2,3$. The scale factors $A_{i}$ must be such that the shear anisotropy is $O(\epsilon)$, where $\epsilon$ is a smallness parameter:

$$
H_{i} \equiv \frac{\dot{A}_{i}}{A_{i}}=H+O(\epsilon), \quad H=\frac{\dot{A}}{A}, \quad A^{3}=A_{1} A_{2} A_{3}
$$

The average expansion is nearly that of a radiation-dominated FRW (spatially flat) universe:

$$
A \sim t^{1 / 2}+O(\epsilon)
$$

As $t \rightarrow 0$, the Weyl curvature hypothesis requires that $H_{i} \rightarrow H$ and $A \rightarrow a t^{1 / 2}(a$ constant).

The classical energy-momentum tensor is assumed to have the perfect fluid radiation form to first order

$$
T_{\mu \nu}=\frac{4}{3} \rho u_{\mu} u_{\nu}+\frac{1}{3} \rho g_{\mu \nu}
$$


where $u^{\mu}=\delta^{\mu}{ }_{0}$ is the preferred four-velocity. This energy-momentum tensor arises from the nearly equilibrium classical photon distribution, which can be expressed in a covariant harmonic expansion in terms of covariant multipoles $F_{\mu \ldots}$ [3]:

$$
f\left(t, k, e^{\mu}\right)=F(t, k)+F_{\mu}(t, k) e^{\mu}+F_{\mu \nu}(t, k) e^{\mu} e^{\nu}+F_{\mu \nu \omega}(t, k) e^{\mu} e^{\nu} e^{\omega}+\cdots
$$

where $k$ is the wave number and $e^{\mu}$ is a unit vector orthogonal to $u^{\mu}$. The monopole moment $F$ is Planckian to first order, while the higher multipoles are $O(\epsilon)$. Only the first three multipoles directly determine $T_{\mu \nu}[3]$ :

$$
\begin{aligned}
\rho & =\frac{4 \pi}{A^{4}} \int_{0}^{\infty} F k^{3} d k \\
q_{\mu} & =\frac{4 \pi}{3 A^{4}} \int_{0}^{\infty} F_{\mu} k^{3} d k \\
\pi_{\mu \nu} & =\frac{8 \pi}{15 A^{4}} \int_{0}^{\infty} F_{\mu \nu} k^{3} d k
\end{aligned}
$$

The energy flux $q_{\mu}$ and anisotropic pressure $\pi_{\mu \nu}$ are zero (to first order) for the energymomentum tensor (4). Although it is possible to achieve this with non-zero $F_{\mu}$ and $F_{\mu \nu}$, it is less artificial and simpler to take

$$
F_{\mu}=0, \quad F_{\mu \nu}=0
$$

The third and higher multipoles, although $O(\epsilon)$, cannot all vanish exactly, since this would force the shear anisotropy to vanish and the geometry to reduce to FRW [4]. With these conditions, (5) is a collision-dominated equilibrium distribution to zero order. It satisfies the Boltzmann equation

$$
p^{\mu} \frac{\partial f}{\partial x^{\mu}}-\Gamma_{\nu \omega}^{\mu} p^{\nu} p^{\omega} \frac{\partial f}{\partial p^{\mu}}=C[f]=c_{\mu} e^{\mu}+\cdots
$$

where $p^{\mu}$ is the four-momentum, and the collision term $C[f]$ vanishes to zero order, so that its multipoles $c_{\mu}, \cdots$ are $O(\epsilon)$.

\section{$2 \quad$ A specific model}

\subsection{Evolution of the universe}

To satisfy (2) and (3), we choose two times $\tau, T$ such that the anisotropy is greatest during $\tau<t<T$. This could correspond to a period where one species decouples 
from the thermalised radiation. Alternatively, we could postulate that this anisotropy interval is imprinted by initial conditions at the singularity. In any case, we choose an evolution of the scale factors according to the ansatz

$$
A_{i}(t)=a t^{1 / 2}\left[\frac{1+\left(\frac{t}{\tau}\right)^{q}}{1+\left(\frac{t}{T}\right)^{q}}\right]^{\frac{\alpha_{i}-1 / 2}{q}}
$$

where $q>1$ and $\left|\alpha_{i}-\frac{1}{2}\right| \ll 1$. Then

$$
A=a t^{1 / 2}\left[\frac{1+\left(\frac{t}{\tau}\right)^{q}}{1+\left(\frac{t}{T}\right)^{q}}\right]^{\frac{\alpha-3 / 2}{3 q}}
$$

where $\alpha \equiv \alpha_{1}+\alpha_{2}+\alpha_{3}$, so that $\left|\alpha-\frac{3}{2}\right| \ll 1$. If we choose $\alpha=\frac{3}{2}$, then $A=a t^{1 / 2}$ and the average scale factor and average rate of expansion are exactly those of FRW.

The rates of expansion given by (11) are

$$
\begin{aligned}
H_{i} & =\frac{1}{2 t}+\left(\alpha_{i}-\frac{1}{2}\right) h(t) \\
H & =\frac{1}{2 t}+\left(\alpha-\frac{3}{2}\right) h(t)
\end{aligned}
$$

where

$$
h(t)=t^{q-1}\left[\frac{T^{q}-\tau^{q}}{\left(t^{q}+\tau^{q}\right)\left(t^{q}+T^{q}\right)}\right]
$$

For simplicity, we assume axisymmetry

$$
A_{2}=A_{1}
$$

and take

$$
\alpha=\frac{3}{2}
$$

Define

$$
\epsilon=\alpha_{3}-\alpha_{1}
$$

as the anisotropy parameter. Then the shear anisotropy may be measured by

$$
\Delta H(t) \equiv H_{3}-H_{1}=\epsilon h(t)
$$


Thus $\Delta H \sim t^{q-1}$ for $t \ll \tau$, while $\Delta H \sim t^{-(q+1)}$ for $t \gg T$. The anisotropy is strongly suppressed towards the singularity and after the anisotropy interval.

The behaviour of the Weyl curvature is given by

$$
C_{\mu \nu \omega \sigma} C^{\mu \nu \omega \sigma}=\frac{1}{72}\left(\frac{d}{d t} \Delta H+H \Delta H\right)^{2} \sim t^{2(q-2)} \quad \text { as } t \rightarrow 0
$$

on using (13), (15) and the results of [5]. Thus the Weyl curvature hypothesis requires $q>2$ [6]. It is shown in [6] that only for $q>2$ is it possible to choose a welldefined initial vacuum state (i.e. without particles) for matter/ radiation fields at the cosmological singularity $t=0$. Therefore the damping of shear anisotropy near the singularity, which is consistent with the (strong) Weyl curvature hypothesis, allows one to choose the initial vacuum state, and to prevent instability of the vacuum for massless conformal fields and infinite creation of particles in the vicinity of the singularity. The expansion law (11) thus makes the singularity stable in relation to production of conformal massless particles. (In fact it is also stable for non-conformal particle creation [6].) Henceforth we take

$$
q=3
$$

\subsection{Quantum field and vacuum definition}

As a model of matter we consider an electromagnetic field in the metric (1). The classical theory is described in [7]. The field equations and secondary quantization were studied in [8]. The time-dependent part of both transversal components of the electromagnetic field with $A_{2}=A_{1}$ satisfies [8]

$$
\frac{d^{2}}{d \eta^{2}} Y(\eta, k)+\left[\frac{2}{3} A \Delta H\right] \frac{d}{d \eta} Y(\eta, k)+k^{2} \delta^{2} Y(\eta, k)=0
$$

where $\eta$ is conformal time $(d t=A d \eta)$ and

$$
\delta^{2}=\sin ^{2} \theta\left(\frac{A_{3}}{A_{1}}\right)^{2 / 3}+\cos ^{2} \theta\left(\frac{A_{1}}{A_{3}}\right)^{4 / 3}
$$

so that by $(11)$

$$
\delta=1+\epsilon \delta_{(1)}+O\left(\epsilon^{2}\right), \quad \delta_{(1)}=\frac{1}{3}\left(1-3 \cos ^{2} \theta\right) \log \left[\frac{1+\left(\frac{t}{\tau}\right)^{3}}{1+\left(\frac{t}{T}\right)^{3}}\right]
$$


If $\beta$ satisfies

$$
\frac{d \beta}{d \eta}+\left(\frac{1}{3} A \Delta H\right) \beta=0
$$

then by $([17)$, the rescaled field $y=Y / \beta$ satisfies

$$
\frac{d^{2} y}{d \eta^{2}}+\left(k^{2} \delta^{2}+m_{e f f}^{2}\right) y=0
$$

where the effective mass of the electromagnetic field is

$$
m_{\text {eff }}^{2}=-\frac{d}{d \eta}\left(\frac{1}{3} A \Delta H\right)-\left(\frac{1}{3} A \Delta H\right)^{2}
$$

To define the choice of the vacuum state, we introduce the representation

$$
Y=\left(\frac{k}{\delta}\right)^{1 / 2}\left(\frac{A_{1}}{A_{3}}\right)\left(\Phi e_{-}+\Psi e_{+}\right), \quad \frac{d Y}{d \eta}=i k \delta\left(\frac{k}{\delta}\right)^{1 / 2}\left(\frac{A_{1}}{A_{3}}\right)\left(\Phi e_{-}-\Psi e_{+}\right)
$$

where

$$
e_{ \pm}=\exp \left( \pm i \int k \delta d \eta\right)=\exp \left( \pm i \int \frac{k \delta}{A} d t\right)
$$

and $\Phi, \Psi$ satisfy

$$
\frac{d \Phi}{d \eta}=\frac{1}{2} W \Psi e_{+}^{2}, \quad \frac{d \Psi}{d \eta}=\frac{1}{2} W \Phi e_{-}^{2}
$$

where $W \equiv \Delta H \sin ^{2} \theta$.

It is natural to define the vacuum state at $\eta=\eta_{0}$ by demanding that the positive frequency solutions be those which satisfy $(d Y / d \eta)_{\eta_{0}}=i k Y\left(\eta_{0}\right)$, or equivalently $\Phi\left(\eta_{0}\right)=1$ and $\Psi\left(\eta_{0}\right)=0$. (This is possible since (18) shows that $\delta\left(\eta_{0}\right)=1$.) In the special case of an FRW universe with $\Delta H=0$ we have $m_{e f f}^{2}=0$ and $W=0$ so $\Phi=$ const, $\Psi=$ const, and the vacuum defined in this way will be stable. Thus one can use the isotropic universe as an absolute standard for the number of photons produced in an anisotropic universe. Correspondingly, such a universe would be an absolute standard for the gravitational entropy which is contained in the gravitational field.

\section{The energy-momentum tensor}




\subsection{Vacuum tensor}

To determine the quantum corrections to the components of the energy-momentum tensor produced by the expansion of our model universe, we adopt the approach of $\mathrm{Hu}$ and Parker [9]. For those modes of the field where we can not neglect $m_{\text {eff }}$ we find the effect of parametric amplification and instability of the vacuum accompanied by the creation or annihilation of particles. For modes of the field with $k \gg m_{e f f} A$, we can neglect the effective mass and they satisfy equations which have effectively isotropic form. For these modes there will be no quantum particle creation. At the early times when photon production is significant, $m_{e f f} \sim 1 / t$, and the critical momentum may be taken as $k_{H}(t) \equiv A(t) / t$, corresponding to wavelengths of the order of the size of the particle horizon.

We separate the energy-momentum tensor of the electromagnetic field into a vacuum polarisation term and a term arising from higher frequency modes which can be treated classically. It is these latter which can be thought of as 'real' photons. They correspond to modes which were parametrically amplified when their wavelength was of the order of the particle horizon or longer $\left(k \leq k_{H}(t)\right)$, when quantum effects were significant to them, but which have since passed within the particle horizon and decoupled from the background thereby forming a classical remnant.

Under the sudden approximation valid for such long wavelength modes, the energymomentum tensor of photons created on scales longer than the particle horizon and then flowing across it and accumulating inside can be written as (compare with [9, 10])

$$
T_{\mu \nu}^{C}(t)=\int_{0}^{t}\left[\frac{A\left(t^{\prime}\right)}{A(t)}\right]^{4} \frac{\partial}{\partial k_{H}} T_{\mu \nu}^{Q}\left(k_{H}\left(t^{\prime}\right), t^{\prime}\right)\left[-\frac{d k_{H}\left(t^{\prime}\right)}{d t^{\prime}}\right] d t^{\prime}
$$

Notice that we use the approximation that no particles are created with $k>k_{H}$, and in (23) we do not include the contribution from all particles created at time $t^{\prime}$, but only those that enter the horizon, because we only take the partial derivative of $n_{Q}$ and $\rho_{Q}$ with respect to $k_{H}$. In $(23), T_{\mu \nu}^{Q}$ is the vacuum expectation value of the energy-momentum tensor operator for the massless field:

$$
T_{\mu \nu}^{Q}(t)=\left\langle 0_{t_{i n}}\left|N_{t} \widehat{T}_{\mu \nu}(t, \vec{x})\right| 0_{t_{i n}}\right\rangle, \quad N_{t} \widehat{T}_{\mu \nu}=\widehat{T}_{\mu \nu}-\left\langle 0_{t}\left|\widehat{T}_{\mu \nu}\right| 0_{t}\right\rangle
$$

where $N_{t}$ indicates normal ordering at time $t$. Here, the initial vacuum state $\left|0_{t=0}\right\rangle$ is determined in the previous section and the transition to the vacuum state $\left|0_{t}\right\rangle$ at time $t$ is done as usual by Bogoliubov transformations. 
Finally we obtain (see also [10, 11])

$$
T_{\mu \nu}^{C}=\frac{1}{(2 \pi)^{2} A^{4}(t)} \int_{0}^{t} \tau_{\mu \nu}\left(t^{\prime}\right) k_{H}^{3}\left(t^{\prime}\right)\left[-\frac{d k_{H}\left(t^{\prime}\right)}{d t^{\prime}}\right] d t^{\prime}
$$

where

$$
\tau_{\nu}^{\mu}(t)=\int \bar{\tau}_{\nu}^{\mu}(t, \theta) \sin \theta d \theta
$$

and the non-zero components of $\bar{\tau}_{\nu}^{\mu}$ are

$$
\begin{aligned}
& \bar{\tau}_{0}^{0}=4 S \delta \\
& \bar{\tau}_{1}^{1}=\bar{\tau}_{2}^{2}=-(2 S+U) \delta \sin ^{2} \theta \\
& \bar{\tau}_{3}^{3}=2\left(-2 S \cos ^{2} \theta+U \sin ^{2} \theta\right) \delta
\end{aligned}
$$

where

$$
S=|\Psi|^{2}, U=2 \operatorname{Re}\left(\Psi^{*} \Phi e_{+}^{2}\right)
$$

In accordance with the conditions of applicability of the formula (24), we can replace the exponentials $e_{ \pm}$by 1 in (22) [9]. Taking the natural initial conditions $\Phi=1$ and $\Psi=0$, one can obtain $\Psi=\sinh R, \Phi=\cosh R$ where

$$
R=\epsilon r, \quad r(t, \theta) \equiv \frac{1}{2} \sin ^{2} \theta \int_{0}^{t} h\left(t^{\prime}\right) d t^{\prime}
$$

Together with (18), this leads to an expansion in $\epsilon$ :

$$
\bar{\tau}^{\mu \nu}=\bar{\tau}_{(0)}^{\mu \nu}+\epsilon \bar{\tau}_{(1)}^{\mu \nu}+\cdots
$$

where

$$
\begin{aligned}
& \bar{\tau}_{0}^{0}=\left(4 r^{2}\right) \epsilon^{2}+O\left(\epsilon^{3}\right) \\
& \bar{\tau}_{1}^{1}=\bar{\tau}_{2}^{2}=-\left(2 r \sin ^{2} \theta\right) \epsilon-\left(2 r\left[r+\delta_{(1)}\right] \sin ^{2} \theta\right) \epsilon^{2}+O\left(\epsilon^{3}\right) \\
& \bar{\tau}_{3}^{3}=\left(4 r \sin ^{2} \theta\right) \epsilon+\left(4 r\left[\delta_{(1)} \sin ^{2} \theta-r \cos ^{2} \theta\right]\right) \epsilon^{2}+O\left(\epsilon^{3}\right)
\end{aligned}
$$

\subsection{Corrections to the classical tensor}

By (26), (27), (28) and (24) it follows that to first order, quantum corrections to the energy-momentum tensor (因) contribute only a trace-free anisotropic pressure tensor:

$$
T_{\mu \nu}^{T}=T_{\mu \nu}+T_{\mu \nu}^{C}=\frac{4}{3} \rho u_{\mu} u_{\nu}+\frac{1}{3} \rho g_{\mu \nu}+\epsilon \pi_{(1) \mu \nu}+O\left(\epsilon^{2}\right)
$$


where

$$
\pi_{(1) \mu \nu}=Q(t) a_{\mu \nu}, \quad a_{\mu}^{\nu} \equiv \operatorname{diag}(0,1,1,-2)
$$

and $Q$ is determined by (24) with (25), (27).

The energy density is only corrected at second order, and no energy flux occurs at any order, owing to the symmetries of (回). By (8), it follows that the quantum corrections constitute a quadrupole moment contribution $F_{\mu \nu}^{C}$ to the photon distribution, where

$$
\pi_{(1) \mu \nu}=\frac{8 \pi}{15 A^{4}} \int_{0}^{\infty} F_{(1) \mu \nu}^{C} k^{3} d k
$$

This gives rise to a quadrupole anisotropy in the temperature fluctuation of radiation. Using the covariant formalism of [3], we find

$$
\frac{\delta T}{T}(\vec{e})=\left(\frac{15 Q a_{\mu \nu} e^{\mu} e^{\nu}}{8 \rho}\right) \epsilon
$$

(Octopole and higher contributions at first order arise from the classical multipoles $F_{\mu \nu \omega}, \cdots$.)

The dynamics of the quadrupole anisotropy are governed by the Boltzmann equation (10). The covariant monopole, dipole and quadrupole moments of (10) for the distribution (5) (with quantum correction) and the spatially homogeneous metric (1D), follow from the general results of [4]. If we linearise these Boltzmann multipoles, we obtain (compare [3]):

$$
\begin{aligned}
k \dot{F}-H k^{2} \frac{\partial F}{\partial k} & \approx 0 \\
0 & \approx c_{\mu} \\
k \dot{F}_{\mu \nu}^{C}-H k^{2} \frac{\partial F_{\mu \nu}^{C}}{\partial k}-\sigma_{\mu \nu} k^{2} \frac{\partial F}{\partial k} & \approx c_{\mu \nu}
\end{aligned}
$$

where $\sigma_{\mu \nu}$ is the shear anisotropy tensor:

$$
\sigma_{\mu \nu}=-\frac{1}{3} \epsilon h a_{\mu \nu}
$$

The first (monopole) and second (dipole) equations imply the linearised energymomentum conservation equations [3]. The first is satisfied identically (to first order) since $F$ is nearly Planckian, and the second because of spatial homogeneity, which 
also implies that the dipole of the collision term vanishes to first order. The third (quadrupole) equation (33), when integrated, gives, using (6) and (8), an evolution equation for $\pi_{\mu \nu}^{C}$ (compare [3]):

$$
\dot{\pi}_{\mu \nu}^{C}+4 H \pi_{\mu \nu}^{C} \approx-\frac{8}{15} \rho \sigma_{\mu \nu}+\gamma_{\mu \nu}
$$

where

$$
\gamma_{\mu \nu}=\frac{8 \pi}{15 A^{4}} \int_{0}^{\infty} c_{\mu \nu} k^{2} d k
$$

is an average quadrupole collision term. By (30) and (34), $\gamma_{\mu \nu}=\gamma(t) a_{\mu \nu}$, and (35) becomes

$$
\dot{Q}+4 H Q=\frac{8}{45} \rho h+\gamma
$$

Using (13), (14), (3) and (6), and the fact that $c_{\mu \nu}$ will decay rapidly outside the anisotropy interval $\tau<t<T$, we find from (36) that

$$
\begin{aligned}
& t \ll \tau \Rightarrow Q \sim t \\
& t \gg T \Rightarrow Q \sim t^{-5}
\end{aligned}
$$

which shows the form of quadrupole anisotropy decay outside the anisotropy interval. By comparison, the Weyl curvature (16) decays at the same rate near the singularity, while (14) shows that the shear anisotropy decays more rapidly at early times, but less rapidly at late times. 


\section{References}

[1] White M, Scott D and Silk J (1994) Annu. Rev. Astron. Astrophys. 32, 319.

[2] Penrose R (1979) General Relativity: An Einstein Centenary Survey, ed Hawking SW and Israel W (Cambridge: Cambridge University Press).

[3] Maartens R, Ellis GFR and Stoeger WR (1995) Phys. Rev. D 51, 1525 and 5942.

[4] Ellis GFR, Matravers DR and Treciokas R (1983) Ann. Phys. 150, 455 and 487.

[5] Birrell ND and Davies PCW (1982) Quantum fields in curved spacetime (Cambridge: Cambridge University Press).

[6] Nesteruk AV (1994) Class. Quantum Grav. 11, L15.

[7] Sagnotti A and Zwiebach B (1981) Phys. Rev. D 24, 305.

[8] Grib AA and Nesteruk AV (1983) Sov. J. Nucl. Phys. 38, 1357.

[9] Hu BL and Parker L (1977) Phys. Lett. A 63, 217.

[10] Nesteruk AV (1991) Class. Quantum Grav. 8, L 243.

[11] Nesteruk AV (1992) Ann. Fondation Louis de Broglie 17, 175. 